\title{
The Schrödinger Equation with Deng-Fan-Eckart Potential (DFEP): Nikiforov-Uvarov-Functional Analysis (NUFA) Method
}

\author{
E. B. Ettah
}

\section{ABSTRACT}

\begin{abstract}
In this study, the radial part of the Schrödinger equation with the DengFan-Eckart potential (DFEP) is solved analytically by employing the improved Greene and Aldrich approximation to bypass the centrifugal barrier and using the Nikiforov-Uvarov-Functional Analysis method (NUFA). The energy expression and wave function are obtained respectively. The numerical energy spectra for some diatomic molecules have been studied and compared with the findings of earlier studies and it has been found to be in good agreement. The NUFA method used in this study is easy and very less cumbersome compared to other methods that currently exist and it is recommended that researchers in this area adopt this method. The findings of this study will find direct applications in molecular physics.
\end{abstract}

Keywords: bound state, NUFA method, non-relativistic, potential model.
Published Online: October 22, 2021

ISSN: $2684-4451$

DOI : 10.24018/ejphysics.2021.3.5.111

\section{E. B. Ettah*}

Department of Physics, Cross University of Technology, Calabar, Cross River State, Nigeria.

(e-mail: emmanuelettah@gmail.com)

*Corresponding Author

\section{INTRODUCTION}

Solving the Schrödinger equation remains a relevant and interesting task in non-relativistic quantum mechanics. This is because the solution of this equation provides the energy spectrum and wave function which are relevant tools for the description of a quantum system. In view of this, researchers over the years have paid great attention to solve this equation with myriads of potential [1-10]. These solutions with several models have been applied to study several physical systems, example: quantum dots [11], quarks [12], diatomic molecules [13], etc. Amongst the numerous potentials proposed and adopted for studying diatomic molecules is the Deng-FanEckart potential (DFEP) proposed by Ikot et al. [14]. This model is given as:

$$
\begin{aligned}
& V(r)=D_{e}\left(1-\frac{b}{\left(e^{\alpha r}-1\right)}\right)^{2}-\frac{V_{1} e^{-\alpha r}}{\left(1-e^{-\alpha r}\right)}+\frac{V_{2} e^{-\alpha r}}{\left(1-e^{-\alpha r}\right)^{2}} \\
& b=e^{\alpha r_{e}}-1
\end{aligned}
$$

where $r_{e}$ is the molecular bond length, $D_{e}$ is the dissociation energy, $r$ is the inter-nuclear distance, $\alpha$ the range of the potential well, $V_{1}$ and $V_{2}$ are the potential strengths. A number of authors have adopted this model to carry out some interesting studies. For instance, Ikot et al. [14] in a maiden study solved the Dirac equation under spin and pseudo-spin symmetries with Deng-Fan-Eckart potential with Coulomb- like and Yukawa-like tensor interaction terms using the Nikiforov-Uvarov method [14]. Edet et al. [15] applied the model to study the diatomic molecules and its thermal properties. Amadi et al. [15] applied this model study the information entropies for $\mathrm{H}_{2}$ and $\mathrm{ScF}$ diatomic molecules.

A number of authors have solved the SE with various potentials and with applications to various physical systems [16]-[20]. Ikot et al. [21] studied effects of magnetic and AB fields on the energy spectra and thermo-magnetic properties of the screened Kratzer potential (SKP). Edet and Ikot [22] studied the effects of magnetic and $\mathrm{AB}$ fields on the energy spectra and thermal properties of some diatomic molecules using the Hulthen-Kratzer potential (HKP) model. Nwabuzor et al. [23] studied the impacts of topological defect on the energy spectra and thermal properties of $\mathrm{LiH}, \mathrm{TiC}$ and $\mathrm{I}_{2}$ diatomic molecules. Edet and Ikot [4] studied the effects of magnetic and $\mathrm{AB}$ fields on the energy spectra and thermomagnetic properties of $\mathrm{CO}$ diatomic molecule using screened modified Kratzer. Ushie et al. [25] studied the effects of the deformation parameter on the non-relativisitc energy spectra of Hulthen-quadratic exponential-type potential model for $\mathrm{H}_{2}, \mathrm{HCl}, \mathrm{CO}$ and $\mathrm{LiH}$ diatomic molecules.

In view of the above, this study will focus on the solutions of the SE with DFEP using the NUFA. The results will be compared with that of Edet et al [15] to show the efficiency of this method.

The paper is organized as follows. In section 2, a brief review of the NUFA method is presented. In section 3, the solution of the 3D Schrödinger equation with the DFEP is presented. In section 4 , result is discussed. Finally, a brief 
concluding remark is given in section 5

\section{NU-FunCtional ANALYSIS (NUFA) METHOD}

Ikot et al. [26] proposed the Nikiforov-Uvarov-Functional Analysis method (NUFA) as a simple and elegant method for solving a second order differential equation of the hypergeometric form. The Nikiforov Uvarov (NU) method [27], the parametric NU method [28], and the functional analysis method [29]-[31] were used. This method, like the parametric NU method, is simple and straightforward. The $\mathrm{NU}$ is well-known for solving a second-order differential equation with the form [28].

$$
\psi_{n}^{\prime \prime}(s)+\frac{\tilde{\tau}(s)}{\sigma(s)} \psi_{n}^{\prime}(s)+\frac{\tilde{\sigma}(s)}{\sigma^{2}(s)} \psi_{n}(s)=0
$$

where $\sigma(s)$ and $\tilde{\sigma}(s)$ are polynomials, at most of second degree, and $\tilde{\tau}(s)$ is a first-degree polynomial. Tezcan and Sever [28] latter introduced the parametric form of NU method in the form:

$$
\psi^{\prime \prime}+\frac{\alpha_{1}-\alpha_{2} s}{s\left(1-\alpha_{3} s\right)} \psi^{\prime}+\frac{1}{s^{2}\left(1-\alpha_{3} s\right)^{2}}\left[-\xi_{1} s^{2}+\xi_{2} s-\xi_{3}\right] \psi(s)=0
$$

where $\alpha_{i}$ and $\xi_{i}(i=1,2,3)$ are all parameters. It can be observed in equation ( 3 ) that the differential equation has two singularities at $s \rightarrow 0$ and $s \rightarrow 1$, thus we take the wave function in the form:

$$
\psi(s)=s^{\lambda}(1-s)^{v} f(s)
$$

Substituting equation (4) into equation (3) leads to the following equation:

$$
\begin{aligned}
& s(1-\alpha s) f^{\prime \prime}(s)+\left[\alpha_{1}+2 \lambda-\left(2 \lambda \alpha_{3}+2 v \alpha_{3}+\alpha_{2}\right) s\right] f^{\prime}(s) \\
& -\alpha_{3}\left(\lambda+v+\frac{\alpha_{2}}{\alpha_{3}}-1+\sqrt{\left(\frac{\alpha_{2}}{\alpha_{3}}-1\right)^{2}+\frac{\xi_{1}}{\alpha_{3}}}\right)\left(\lambda+v+\frac{\alpha_{2}}{\alpha_{3}^{2}}-1+\sqrt{\left(\frac{\alpha_{2}}{\alpha_{3}}-1\right)^{2}+\frac{\xi_{1}}{\alpha_{3}^{2}}}\right) \\
& +\left[\frac{\lambda(\lambda-1)+\alpha_{1} \lambda-\xi_{3}}{s}+\frac{\alpha_{2} v-\alpha_{1} \alpha_{3} v+v(v-1) \alpha_{3}-\frac{\xi_{1}}{\alpha_{3}}+\xi_{2}-\xi_{3} \alpha_{3}}{\left(1-\alpha_{3} s\right)}\right] f(s)=0
\end{aligned}
$$

Equation (5) can be reduced to a Gauss hypergeometric equation if and only if the following functions vanished,

$$
\begin{aligned}
& \lambda(\lambda-1)+\alpha_{1} \lambda-\xi=0 \\
& \alpha_{2} v-\alpha_{1} \alpha_{3} v+v(v-1) \alpha_{3}-\frac{\xi_{1}}{\alpha_{3}}+\xi_{2}-\xi_{3} \alpha=0
\end{aligned}
$$

Thus, equation (5) becomes:

$$
\begin{aligned}
& s\left(1-\alpha_{1} s\right) f^{\prime \prime}(s)+\left[\alpha_{1}+2 \lambda-\left(2 \lambda \alpha_{3}+2 v \alpha_{3}+\alpha_{2}\right) s\right] f^{\prime}(s) \\
& -\alpha_{3}\left(\lambda+v+\frac{\alpha_{2}}{\alpha_{3}}-1+\sqrt{\left(\frac{\alpha_{2}}{\alpha_{3}}-1\right)^{2}+\frac{\xi_{1}}{\alpha_{3}}}\right)\left(\lambda+v+\frac{\alpha_{2}}{\alpha_{3}^{2}}-1+\sqrt{\left(\frac{\alpha_{2}}{\alpha_{3}}-1\right)^{2}+\frac{\xi_{1}}{\alpha_{3}^{2}}}\right) f(s)=0
\end{aligned}
$$

Solving equations (6) and (7) completely give:

$$
\lambda=\frac{\left(1-\alpha_{1}\right) \pm \sqrt{\left(1-\alpha_{1}\right)^{2}+4 \xi_{3}}}{2}
$$

$$
v=\frac{\left(\alpha_{3}+\alpha_{1} \alpha_{3}-\alpha_{2}\right) \pm \sqrt{\left(\alpha_{3}+\alpha_{1} \alpha_{3}-\alpha_{2}\right)^{2}+4\left(\frac{\xi_{1}}{\alpha_{3}}+\alpha_{3} \xi_{3}-\xi_{2}\right)}}{2}
$$

Equation (8) is the hypergeometric equation type of the form:

$$
x(1-x) f^{\prime \prime}(x)+[c+(a+b+1) x] f^{\prime}(x)-a b f(x)=0
$$

Using equations (4), (8) and (11), we obtain the energy equation and the corresponding wave equation respectively for the NUFA method as follows:

$$
\begin{aligned}
& \lambda^{2}+2 \lambda\left(v+\frac{\alpha_{2}}{\alpha_{3}}-1+\frac{n}{\sqrt{\alpha_{3}}}\right)+\left(v+\frac{\alpha_{2}}{\alpha_{3}}-1+\frac{n}{\sqrt{\alpha_{3}}}\right)^{2}-\left(\frac{\alpha_{2}}{\alpha_{3}}-1\right)^{2}-\frac{\xi_{1}}{\alpha_{3}^{2}}=0 \\
& \psi(s)=N s^{\frac{\left(1-\alpha_{1}\right)+\sqrt{\left(1-\alpha_{1}\right)^{2}+4 \xi_{3}}}{2}}\left(1-\alpha_{3} s\right) \frac{\left(\alpha_{3}+\alpha_{1} \alpha_{3}-\alpha_{2}\right)+\sqrt{\left(\alpha_{3}+\alpha_{1} \alpha_{3}-\alpha_{2}\right)^{2}+4\left(\frac{\xi_{1}}{\alpha_{3}^{2}+\alpha_{3} \xi_{3}-\xi_{2}}\right)}}{2}{ }_{2} F_{1}(a, b, c ; s)
\end{aligned}
$$

where $a, b, c$ are given as follows:

$$
\begin{aligned}
& a=\sqrt{\alpha_{3}}\left(\lambda+v+\frac{\alpha_{2}}{\alpha_{3}}-1+\sqrt{\left(\frac{\alpha_{2}}{\alpha_{3}}-1\right)^{2}+\frac{\xi_{1}}{\alpha_{3}}}\right) \\
& b=\sqrt{\alpha_{3}}\left(\lambda+v+\frac{\alpha_{2}}{\alpha_{3}}-1-\sqrt{\left(\frac{\alpha_{2}}{\alpha_{3}}-1\right)^{2}+\frac{\xi_{1}}{\alpha_{3}}}\right) \\
& \alpha_{1}+2 \lambda
\end{aligned}
$$

\section{SCHRÖDINGER EQUATION WITH DENG-FAN-ECKART POTENTIAL}

The radial part of the Schrödinger equation is given by [15], [32], [33]:

$$
\frac{d^{2} \psi(r)}{d r^{2}}+\frac{2 \mu}{\hbar^{2}}\left[E_{n \ell}-V(r)-\frac{\hbar^{2} \ell(\ell+1)}{2 \mu r^{2}}\right] \psi(r)=0
$$

Substituting Eq. (1) into Eq. (17), the radial Schrödinger equation is obtained in the following form: 
$\frac{d^{2} \psi(r)}{d r^{2}}+\frac{2 \mu}{\hbar^{2}}\left[\begin{array}{l}E_{n \ell}-\left(D_{e}+\frac{D_{e} b^{2} e^{-2 \alpha r}}{\left(1-e^{-\alpha r}\right)^{2}}-\frac{2 D_{e} b e^{-\alpha r}}{\left(1-e^{-\alpha r}\right)}-\frac{V_{1} e^{-\alpha r}}{\left(1-e^{-\alpha r}\right)}+\frac{V_{2} e^{-\alpha r}}{\left(1-e^{-\alpha r}\right)^{2}}\right) \\ -\frac{\hbar^{2} \ell(\ell+1)}{2 \mu r^{2}}\end{array}\right] \psi(r)=0$

Eq. (18) in its current form cannot be solved with any standard mathematical procedure analytically for $\ell \neq 0$ because of the centrifugal barrier. This calls for a need to adopt and apply an approximation to the centrifugal term. The following approximation is adopted [34], [35]:

$$
\frac{1}{r^{2}}=\alpha^{2}\left[d_{0}+\frac{e^{\alpha r}}{\left(1-e^{\alpha r}\right)^{2}}\right]
$$

Inserting Eqs. (19) into Eq. (18) where we have the $\frac{1}{r^{2}}$ term, we have:

$$
\frac{d^{2} \psi(r)}{d r^{2}}+\frac{2 \mu}{\hbar^{2}}\left[\begin{array}{l}
E_{n e}-\left(D_{e}+\frac{D_{e} b^{2} e^{-2 \alpha r}}{\left(1-e^{-\alpha r}\right)^{2}}-\frac{2 D_{e} b e^{-\alpha r}}{\left(1-e^{-\alpha r}\right)}-\frac{V_{1} e^{-\alpha r}}{\left(1-e^{-\alpha r}\right)}+\frac{V_{2} e^{-\alpha r}}{\left(1-e^{-\alpha r}\right)^{2}}\right) \\
\frac{\hbar^{2} \ell(\ell+1) \alpha^{2}}{2 \mu}\left(d_{0}+\frac{e^{\alpha r}}{\left(1-e^{\alpha r}\right)^{2}}\right)
\end{array}\right] \psi(r)=0
$$

The following dimensionless parameters have been introduced:

$-\varepsilon^{2}=\frac{2 \mu\left(E_{n \ell}-D_{e}\right)}{\hbar^{2} \alpha^{2}}, u_{1}=\frac{2 \mu D_{e} b^{2}}{\hbar^{2} \alpha^{2}}, u_{2}=\frac{4 \mu D_{e} b}{\hbar^{2} \alpha^{2}}, P_{1}=\frac{2 \mu V_{1}}{\hbar^{2} \alpha^{2}}, P_{2}=\frac{2 \mu V_{2}}{\hbar^{2} \alpha^{2}}$ and

$$
\gamma=\ell(\ell+1)
$$

Hence, Eq. (20) can be rewritten in a less ambiguous form as:

$\frac{d^{2} \psi(r)}{d r^{2}}+\frac{2 \mu}{\hbar^{2}}\left[\begin{array}{l}-\varepsilon^{2}\left(1-e^{-\alpha r}\right)^{2}-u_{1} e^{-2 \alpha r}+u_{2} e^{-\alpha r}\left(1-e^{-\alpha r}\right)+P_{1} e^{-\alpha r}\left(1-e^{-\alpha r}\right) \\ -P_{2} e^{-\alpha r}-\gamma e^{-\alpha r}-\gamma d_{0}\left(1-e^{-\alpha r}\right)^{2}\end{array}\right] \psi(r)=0$

The transformation $t=e^{-\alpha r}$ is utilized and it transforms Eq. (22) to the hypergeometric-type of the form:

$$
\frac{d^{2} \psi(t)}{d t^{2}}+\frac{1}{t} \frac{d \psi(t)}{d t}+\frac{1}{t^{2}(1-t)^{2}}\left[\begin{array}{l}
-\left(\varepsilon^{2}+u_{1}+u_{2}+P_{1}+\gamma d_{0}\right) t^{2} \\
+\left(2 \varepsilon^{2}+u_{2}+P_{1}-P_{2}-\gamma+2 \gamma d_{0}\right) t-\left(\varepsilon^{2}+\gamma d_{0}\right)
\end{array}\right] \psi(t)=0
$$

Comparing (23) and (3), we obtain the following:

$$
\begin{aligned}
& \alpha_{1}=\alpha_{2}=\alpha_{3}=1, \\
& \lambda=\sqrt{\varepsilon^{2}+\gamma d_{0}} \\
& \nu=\frac{1}{2}+\sqrt{\frac{1}{4}+u_{1}+P_{2}+\gamma}
\end{aligned}
$$

The energy is then obtained from (12) as follows:

$$
(\lambda+v)-\sqrt{\varepsilon^{2}+u_{1}+u_{2}+P_{1}+\gamma d_{0}}=-n \quad n=0,1,2 \ldots
$$

from which we obtain:

$$
\varepsilon^{2}=-\gamma d_{0}+\frac{1}{4}\left(\frac{\left(n+\frac{1}{2}+\sqrt{\frac{1}{4}+u_{1}+P_{2}+\gamma}\right)^{2}-u_{1}-u_{2}-P_{1}}{\left(n+\frac{1}{2}+\sqrt{\frac{1}{4}+u_{1}+P_{2}+\gamma}\right)}\right)^{2}
$$

On substitution of the value of the dimensionless parameters in Eq. (21) into Eq. (26), the solutions is obtained as follows:

$$
E_{n \ell}=D_{e}+\frac{\hbar^{2} \alpha^{2} \ell(\ell+1) d_{0}}{2 \mu}-\frac{\hbar^{2} \alpha^{2}}{2 \mu}\left(\frac{(n+v)^{2}-\frac{2 \mu D_{e} b^{2}}{\hbar^{2} \alpha^{2}}-\frac{4 \mu D_{e} b}{\hbar^{2} \alpha^{2}}-\frac{2 \mu V_{1}}{\hbar^{2} \alpha^{2}}}{2(n+v)}\right)^{2}
$$

where

$$
v=\frac{1}{2}+\frac{1}{2} \sqrt{(2 \ell+1)^{2}+\frac{8 \mu D_{e} b^{2}}{\hbar^{2} \alpha^{2}}+\frac{8 \mu V_{2}}{\hbar^{2} \alpha^{2}}}
$$

Similarly, the wave function is obtained as:

$$
\psi(t)=N_{n \ell} t^{\sqrt{\varepsilon^{2}+\gamma d_{0}}}(1-t)^{\frac{1}{2}+\sqrt{\frac{1}{4}+u_{1}+P_{2}+\gamma}}{ }_{2} F_{1}(-n, n+2(\lambda+v) ; 2 \lambda+1 ; t)
$$

\section{Discussion OF RESUlts}

In order to show the validity of the results obtained in this study, the numerical energy levels is computed by utilizing Eq. (27) for different quantum numbers $n$ and $\ell$ with for four Diatomic molecules ( $\mathrm{H}_{2}, \mathrm{CO}, \mathrm{ScN}$ and $\mathrm{ScF}$ ). To achieve this objective, spectroscopic parameters shown in Table I are adopted from the NIST database and the following conversions:

$$
\begin{aligned}
& 1 \mathrm{amu}=931.494028 \mathrm{MeV} / \mathrm{c}^{2}, \\
& 1 \mathrm{~cm}^{-1}=1.239841875 \times 10^{-4} \mathrm{eV} \AA \text { are used [15]. }
\end{aligned}
$$

The numerical energy values obtained by adopting the experimental fitting parameters are presented in Table II and Table III for several vibrational and rotational quantum numbers. To firmly establish the validity and accuracy of our results, the numerical energy is compared with the results of Edet et al. [15] and from observation, it is clearly seen that there is an excellent agreement. 
TABLE I: SPECTROSCOPIC PARAMETERS OF THE MOLECULES USED IN THIS WORK

\begin{tabular}{ccccc}
\hline Parameters & $H_{2}[15]$ & $C O[15]$ & $S c N[15]$ & $S c F[15]$ \\
\hline$D_{e}\left(\mathrm{~cm}^{-1}\right)$ & 38266 & 90540 & 36778.88 & 47183.43 \\
$r_{e}(\AA)$ & 0.7416 & 1.1283 & 1.768 & 1.794 \\
$\alpha\left(\AA^{-1}\right)$ & 1.9426 & 2.294 & 1.5068 & 1.46102 \\
$\mu($ a.m.u $)$ & 0.50391 & 6.860672 & 10.68277 & 13.35894 \\
\hline
\end{tabular}

TABLE II: COMPARISON OF BOUND STATE ENERGIES $E_{n \ell}(e \mathrm{~V})$ FOR $H_{2}$

AND $C O$ MOLECULES OBTAINED BY USING NiKIFOROV-UvaROV-

FUNCTIONAL ANALYSIS METHOD (NUFA) WITH FACTORIZATION METHOD FOR DIFFERENT VALUES OF THE $N$ AND $\ell$ QUANTUM NUMBERS

\begin{tabular}{cccccc}
\hline$n$ & $\ell$ & $\begin{array}{c}H_{2} \\
(\mathrm{NUFA})\end{array}$ & $\begin{array}{c}\mathrm{H}_{2} \\
(\mathrm{FAA})[1]\end{array}$ & $\mathrm{CO}$ (NUFA) & $C O$ (FAA) [1] \\
\hline 0 & 0 & 0.3161089 & 0.3161089 & 0.3653484 & 0.3653484 \\
0 & 1 & 0.3304972 & 0.3304972 & 0.3658917 & 0.3658917 \\
\hline 1 & 0 & 0.9626475 & 0.9626475 & 0.6487759 & 0.6487759 \\
1 & 1 & 0.9759181 & 0.9759181 & 0.6493153 & 0.6493153 \\
\hline 2 & 0 & 1.5466492 & 1.5466492 & 0.9281700 & 0.9281700 \\
2 & 1 & 1.5588663 & 1.5588663 & 0.9287056 & 0.9287056 \\
2 & 2 & 1.5832386 & 1.5832386 & 0.9297766 & 0.9297766 \\
\hline 3 & 0 & 2.0716668 & 2.0716668 & 1.2035434 & 1.2035434 \\
3 & 1 & 2.0828895 & 2.0828895 & 1.2040751 & 1.2040751 \\
3 & 2 & 2.1052768 & 2.1052768 & 1.2051383 & 1.2051383 \\
3 & 3 & 2.1387131 & 2.1387131 & 1.2067333 & 1.2067333 \\
\hline 4 & 0 & 2.5409692 & 2.5409692 & 1.4749081 & 1.4749081 \\
4 & 1 & 2.5512519 & 2.5512519 & 1.4754360 & 1.4754360 \\
4 & 2 & 2.5717628 & 2.5717628 & 1.4764914 & 1.4764914 \\
4 & 3 & 2.6023934 & 2.6023934 & 1.4780750 & 1.4780750 \\
4 & 4 & 2.6429822 & 2.6429822 & 1.4801861 & 1.4801861 \\
\hline 5 & 0 & 2.9575685 & 2.9575685 & 1.7422767 & 1.7422767 \\
5 & 1 & 2.9669614 & 2.9669614 & 1.7428007 & 1.7428007 \\
5 & 2 & 2.9856959 & 2.9856959 & 1.7438485 & 1.7438485 \\
5 & 3 & 3.0136703 & 3.0136703 & 1.7454204 & 1.7454204 \\
5 & 4 & 3.0507328 & 3.0507328 & 1.7475162 & 1.7475162 \\
5 & 5 & 3.0966829 & 3.0966829 & 1.7501360 & 1.7501360 \\
\hline & & & & &
\end{tabular}

TABLE III: COMPARISON OF BOUND STATE ENERGIES $E_{n \ell}(e \mathrm{~V})$ FOR $S c N$

AND $S c F$ MOLECULES OBTAINED BY USING NIKIFOROV-UVAROV-

FUNCTIONAL ANALYSIS METHOD (NUFA) WITH FACTORIZATION METHOD FOR DIFFERENT VALUES OF THE $N$ AND $\ell$ QUANTUM NUMBERS

\begin{tabular}{llcccc}
\hline$n$ & $\ell$ & $\begin{array}{c}S c N \\
\text { (NUFA) }\end{array}$ & $\begin{array}{c}S c N(\mathrm{FAA}) \\
{[1]}\end{array}$ & $\begin{array}{c}S c F \\
(\mathrm{NUFA})\end{array}$ & $\begin{array}{c}S F \text { (FAA) } \\
{[1]}\end{array}$ \\
\hline 0 & 0 & 0.5678234 & 0.5678234 & 0.7807499 & 0.7807499 \\
0 & 1 & 0.5679634 & 0.5679634 & 0.7808573 & 0.7808573 \\
\hline 1 & 0 & 0.6573524 & 0.6573524 & 0.8685782 & 0.8685782 \\
1 & 1 & 0.6574917 & 0.6574917 & 0.8686851 & 0.8686851 \\
\hline 2 & 0 & 0.7457977 & 0.7457977 & 0.9555848 & 0.9555848 \\
2 & 1 & 0.7459361 & 0.7459361 & 0.9556913 & 0.9556913 \\
2 & 2 & 0.7462130 & 0.7462130 & 0.9559042 & 0.9559042 \\
\hline 3 & 0 & 0.8331616 & 0.8331616 & 1.0417712 & 1.0417712 \\
3 & 1 & 0.8332993 & 0.8332993 & 1.0418772 & 1.0418772 \\
3 & 2 & 0.8335745 & 0.8335745 & 1.0420891 & 1.0420891 \\
3 & 3 & 0.8339876 & 0.8339876 & 1.0424072 & 1.0424072 \\
\hline 4 & 0 & 0.9194467 & 0.9194467 & 1.1271390 & 1.1271390 \\
4 & 1 & 0.9195836 & 0.9195836 & 1.1272445 & 1.1272445 \\
4 & 2 & 0.9198573 & 0.9198573 & 1.1274555 & 1.1274555 \\
4 & 3 & 0.9202679 & 0.9202679 & 1.1277721 & 1.1277721 \\
4 & 4 & 0.9208153 & 0.9208153 & 1.1281941 & 1.1281941 \\
\hline 5 & 0 & 1.0046555 & 1.0046555 & 1.2116896 & 1.2116896 \\
5 & 1 & 1.0047916 & 1.0047916 & 1.2117947 & 1.2117947 \\
5 & 2 & 1.0050637 & 1.0050637 & 1.2120047 & 1.2120047 \\
5 & 3 & 1.0054720 & 1.0054720 & 1.2123199 & 1.2123199 \\
5 & 4 & 1.0060162 & 1.0060162 & 1.2127400 & 1.2127400 \\
5 & 5 & 1.0066966 & 1.0066966 & 1.2132652 & 1.2132652 \\
\hline & & & & & \\
& & & & & \\
\hline
\end{tabular}

\section{CONCLUSION}

In this study, the three-dimensional Schrodinger equation with the Deng-Fan-Eckart potential (DFEP) is solved using the Nikiforov-Uvarov functional analysis (NUFA) method.
The energy equation and wave function are obtained respectively. The numerical energy spectra for some diatomic molecules have been studied and compared with the findings of Edet et al. [15] and it has been found to be in perfect agreement. However, it is interesting to point out here that the NUFA method used in this study is easy and very less cumbersome compared the other methods that currently exist. The findings of this study will find direct applications in molecular physics.

\section{REFERENCES}

[1] H. Louis, I. B. Iserom, M. T. Odey, A. U. Ozioma, N. I. Nelson, I. I Alexander, A. I. Pigweh and E. C. Okon, "Solutions to the Dirac equation for Manning-Rosen plus shifted Deng-Fan potential and Coulomb-like tensor interaction using Nikiforov-Uvarov method," International Journal of Chemistry, vol. 10, pp. 99-106, 2018 https://doi.org/10.5539/ijc.v10n3p99.

[2] H. Louis, I. Iserom, O. U. Akakuru, N. A. Nzeala-ibe, A. I. Ikeuba, T. O. Magu, A. I. Pigweh and E. O. Collins 1-state solutions of the relativistic and non-relativistic wave equations for modified HylleraasHulthen potential using the Nikiforov-Uvarov quantum formalism. Oriental J. Phys. Sci, vol. 3, pp. 3, 2018 http://www.orientjphysicalsciesnces.org/.

[3] J. E. Ntibi., E. P. Inyang, E. P. Inyang, and E. S. William, "Relativistic Treatment of D-Dimensional Klien-Gordon equation with Yukawa potential," International Journal of Innovative Science, Engineering Technology, vol. 11, pp. 2348-7968, 2020.

[4] C. O. Edet and P. O. Okoi, "Any 1-state solutions of the Schrödinger equation for q-deformed Hulthen plus generalized inverse quadratic Yukawa potential in arbitrary dimensions," Revista mexicana de física, vol. 65, pp. 333-344, 2019. https://doi.org/10.31349/revmexfis.65.333.

[5] C. O. Edet, U. S. Okorie, A. T. Ngiangia, and A. N. Ikot, "Bound state solutions of the Schrodinger equation for the modified Kratzer potential plus screened Coulomb potential," Indian journal of Physics, vol. 94 pp. 425-433, 2020. https://doi.org/10.1007/s12648-019-01477-9.

[6] C. O. Edet, K. O. Okorie, H. Louis and N. A. Nzeata-Ibe, "Any l-state solutions of the Schrodinger equation interacting with HellmannKratzer potential model," Indian Journal of Physics, vol. 94, pp. 243 251, 2020. https://doi.org/10.1007/s12648-019-01467-x.

[7] C. S. Jia, J. Y. Wang, S. He, and L. T. Sun, "Shape invariance and the supersymmetry WKB approximation for a diatomic molecule potential," Journal of Physics A: Mathematical and General, vol. 33, pp. 6993, 2000. https://doi.org/10.1088/0305-4470/33/39/313.

[8] C. O. Edet, P. O. Okoi and S. O. Chima, "Analytic solutions of the Schrödinger equation with non-central generalized inverse quadratic Yukawa potential," Revista Brasileira de Ensino de Física, vol. 42, pp. e20190083, 2020. https://doi.org/10.1590/1806-9126-RBEF-2019. 0083.

[9] P. O. Okoi, C. O. Edet, and T. O. Magu, "Relativistic treatment of the Hellmann-generalized Morse potential," Revista mexicana de fisica, vol. 66, pp. 1-13, 2020. https://doi.org/10.31349/RevMexFis.66.1.

[10] R. Khordad, H. Bahramiyan and H. R. Rastegar Sedehi, "Effects of strain, magnetic field and temperature on entropy of a two dimensional GaAs quantum dot under spin-orbit interaction," Optical and Quantum Electronics, vol. 50, pp. 294, 2018. https://doi.org/10.1007/s11082018-1557-2.

[11] E. P. Inyang, I. O. Akpan, J. E. Ntibi and E. S. William, “Analytical solutions of the Schrödinger equation with class of Yukawa potential for a Quarkonium system via series expansion method," European Journal of Applied Physics, vol. 2, pp. 1-6, 2020.

[12] A. N. Ikot, U. Okorie, A. T. Ngiangia, C. A. Onate, C. O. Edet, I. O. Akpan and P. O. Amadi, "Bound state solutions of the Schrödinger equation with energy-dependent molecular Kratzer potential via asymptotic iteration method," Ecletica Quimica Journal, vol. 45, pp 65-76, 2020.https://doi.org/10.26850/1678-4618eqj.v45.1.p65-76.

[13] A. N. Ikot, S. Zarrinkamar, B. H. Yazarloo, H. Hassanabadi, "Relativistic symmetries of Deng Fan and Eckart potentials with Coulomb-like and Yukawa-like tensor interactions," Chinese Journal of Physical, vol. 23, pp. 100306, 2014. https://doi.org/10.1088/16741056/23/10/100306.

[14] C. O. Edet, U. S. Okorie, G. Osobonye, A. N. Ikot, G. J. Rampho, and R. Sever, "Thermal properties of Deng-Fan-Eckart potential model using Poisson," summation approach, Journal Mathematical Chemistry, vol. 58, pp. 989, 2020. https://doi.org/10.1007/s10910-02001107-4. 
[15] P. O. Amadi, A. N. Ikot, G. J. Rampho, U. S. Okorie, H. Y. Abdullah and B. C. L"utf"uo־glu, "Information entropies for $\mathrm{H} 2$ and ScF diatomic molecules with Deng- Fan-Eckart potential," Revista Mexicana de F'lsica, vol. 66, pp. 742-748, 2021. https://doi.org/10.31349/RevMexFis.66.742.

[16] G. J. Rampho, A. N. Ikot, C. O. Edet, and Okorie, "Energy spectra and thermal properties of diatomic molecules in the presence of magnetic and AB fields with improved Kratzer potential," Molecular Physics, vol. 119, pp. 2021 https://doi.org/10.1080/00268976.2020.1821922.

[17] C. O. Edet, A. N. Ikot, M. C. Onyeaju, U. S. Okorie, G. J. Rampho, M. L. Lekala, and S. Kaya, "Thermo-magnetic properties of the screened Kratzer potential with spatially varying mass under the influence of Aharanov-Bohm (AB) and position-dependent magnetic fields," Physica E: Low-dimensional Systems and Nanostructures, vol. 131, pp. 114710, 2021. https://doi.org/10.1016/j.physe.2021.114710.

[18] A. N. Ikot, C. O. Edet, U. S. Okorie, A-H. Abdel-Aty, M Ramantswana, G. J. Rampho, N. A. Alshehri, S. K. Elagan and S. Kaya, "Solutions of the 2D Schrodinger equation and its thermal properties for improved ultra-generalized exponential hyperbolic potential (IUGE-HP)," The European Physical Journal Plus, vol. 136, pp. 1-18, 2021. https://doi.org/10.1140/epjp/s13360-021-01408-w.

[19] C. O. Edet, A. N. Ikot, U. S. Okorie, G. J. Rampho, M. Ramantswana, R. Horchani, H. Abdullah, J. A. Vinasco, C. A. Duque and A-H. Abdel-Aty, "Persistent Current, Magnetic Susceptibility, and Thermal Properties for a Class of Yukawa Potential in the Presence of External Magnetic and Aharanov-Bohm Fields," International Journal of Thermophysics, vol. 42, pp. 1-18, 2021. https://doi.org/10.1007/s10765-021-02891-0.

[20] A. N. Ikot, C. O. Edet, P.O. Amadi, U. S. Okorie, G. J. Rampho, and H. Y. Abdullah, "Thermodynamic properties of Aharanov-Bohm (AB) and magnetic fields with screened Kratzer potential," The European Physical Journal D, vol. 74, pp. 1-13, 2020. https://doi.org/10.1140/epjd/e2020-10084-9.

[21] C. O. Edet, and A. N. Ikot, Analysis of the impact of external fields on the energy spectra and thermo-magnetic properties of $\mathrm{N}_{2}, \mathrm{I}_{2}, \mathrm{CO}, \mathrm{NO}$ and $\mathrm{HCl}$ diatomic molecules. Molecular Physics, pp. e1957170, 2021. https://doi.org/10.1080/00268976.2021.1957170.

[22] P. Nwabuzor, C. Edet, A. N. Ikot, U. Okorie, M. Ramantswana, R. Horchani, A-H. Abdel-Aty and G.Rampho. "Analyzing the Effects of Topological Defect (TD) on the Energy Spectra and Thermal Properties of $\mathrm{LiH}, \mathrm{TiC}$ and $\mathrm{I}_{2}$ Diatomic Molecules" Entropy, 23, 1060, 2021. https://doi.org/10.3390/e23081060.

[23] C. O. Edet and A. N. Ikot, "Effects of Topological Defect on the Energy Spectra and Thermo-magnetic Properties of $\$ \$$ CO $\$ \$$ CO Diatomic Molecule" Journal of Low Temperature Physics, vol. 203, pp.84-111, 2021.https://doi.org/10.1007/s10909-021-02577-9.

[24] U. P. Obogo, O. E. Ubi, C. O. Edet and A. N. Ikot, "Effect of the deformation parameter on the nonrelativistic energy spectra of the qdeformed Hulthen-quadratic exponential-type potential," Eclética Química Journal, vol. 46, pp. 60-73, 2021. https://doi.org/10.26850/1678-4618eqj.v46.4.2021.p60-73.

[25] A. N. Ikot, U. S. Okorie, P. O. Amadi, C. O. Edet, G. J. Rampho and R. Sever, "The Nikiforov-Uvarov-Functional Analysis (NUFA) Method: A New Approach for Solving Exponential-Type Potentials," Few-Body Systems, vol. 62, pp. 1-16, 2021. https://doi.org/10.1007/s00601-021-01593-5.

[26] A. F. Nikiforov and V. B. Uvarov (1988). Special functions of mathematical physics (Vol. 205). Basel: Birkhäuser.

[27] C. Tezcan and R. Sever, "A general approach for the exact solution of the Schrödinger equation," International Journal of Theoretical Physics, vol. 48, pp. 337-350, 2009.

[28] S. H. Dong (2007). Factorization method in quantum mechanics (Vol. 150). Springer Science \& Business Media.

[29] C. O. Edet, P. O. Amadi, M. C. Onyeaju, U. S. Okorie, R. Sever, G. J. Rampho, H. Y. Abdullah, I. H. Salih and A. N. Ikot, "Thermal Properties and Magnetic Susceptibility of Hellmann Potential in Aharonov-Bohm (AB) Flux and Magnetic Fields at Zero and Finite Temperatures. Journal of Low Temperature Physics, vol. 202, pp. 83105, 2021. https://doi.org/10.1007/s10909-020-02533-z.

[30] U. S. Okorie, A. N. Ikot, C. O. Edet, I. O. Akpan, R. Sever and G. J. Rampho, "Solutions of the Klein Gordon equation with generalized hyperbolic potential in D-dimensions," Journal of Physics Communications, vol. 3, pp. 095015, 2019. https://doi.org/10.1088/2399-6528/ab42c6.

[31] C. O. Edet, P. O. Amadi, U. S. Okorie, A. Tas, A. N. Ikot and G. J. Rampho, "Solutions of Schrodinger equation and thermal properties of generalized trigonometric Poschl-Teller potential," Revista Mexicana de Física, vol. 66, pp. 824-839, 2020 https://doi.org/10.31349/RevMexFis.66.824.
[32] C. Edet, "Effects of Magnetic and Aharanov-Bohm (AB) Fields on the Energy Spectra of the Yukawa Potential," 2020 arXiv preprint arXiv:2012.08644.

[33] R. L. Greene and C. Aldrich, "Variational wave functions for a screened Coulomb potential," Physical Review A, vol. 14, pp. 2363, 1976.

[34] B. I. Ita, H. Louis, O. U. Akakuru, N. A. Nzeata-Ibe, A. I. Ikeuba, T. O. Magu, P. O. Amos and C. O. Edet, "Approximate Solution to the Schrödinger Equation with Manning-Rosen plus a Class of Yukawa Potential via WKBJ Approximation Method,” Bulg. J. Phys, vol. 45, pp. 323-333, 2018. https://www.bjp-bg.com/paper1.php?id=914. 